\title{
A TYCHONOFF THEOREM IN INTUITIONISTIC FUZZY TOPOLOGICAL SPACES
}

\author{
DOǦAN ÇOKER, A. HAYDAR EŞ, and NECLA TURANLI
}

Received 23 March 2004 and in revised form 6 October 2004

In memory of Professor Dr. Doğan Çoker

\begin{abstract}
The purpose of this paper is to prove a Tychonoff theorem in the so-called "intuitionistic fuzzy topological spaces." After giving the fundamental definitions, such as the definitions of intuitionistic fuzzy set, intuitionistic fuzzy topology, intuitionistic fuzzy topological space, fuzzy continuity, fuzzy compactness, and fuzzy dicompactness, we obtain several preservation properties and some characterizations concerning fuzzy compactness. Lastly we give a Tychonoff-like theorem.
\end{abstract}

2000 Mathematics Subject Classification: 03F55, 54A40.

1. Introduction. After the introduction of the concept of fuzzy sets by Zadeh [8] several researches were conducted on the generalizations of the notion of fuzzy set. The idea of intuitionistic fuzzy set (IFS) was first published by Atanassov [1] and many works by the same author and his colleagues appeared in the literature [1, 3, 4].

Later, this concept was generalized to "intuitionistic $L$-fuzzy sets" by Atanassov and Stoeva [4].

2. Preliminaries. First we will present the fundamental definitions obtained by Atanassov.

DeFINITION 2.1 [1]. Let $X$ be a nonempty fixed set. An IFS $A$ is an object having the form

$$
A=\left\{\left\langle x, \mu_{A}(x), \gamma_{A}(x)\right\rangle: x \in X\right\},
$$

where the functions $\mu_{A}: X \rightarrow I$ and $\gamma_{A}: X \rightarrow I$ denote the degree of membership (namely, $\mu_{A}(x)$ ) and the degree of nonmembership (namely, $\gamma_{A}(x)$ ) of each element $x \in X$ to the set $A$, respectively, and $0 \leq \mu_{A}(x)+\gamma_{A}(x) \leq 1$ for each $x \in X$.

REMARK 2.2. An IFS $A=\left\{\left\langle x, \mu_{A}(x), \gamma_{A}(x)\right\rangle: x \in X\right\}$ in $X$ can be identified to an ordered pair $\left\langle\mu_{A}, \gamma_{A}\right\rangle$ in $I^{X} \times I^{X}$ or to an element in $(I \times I)^{X}$.

REMARK 2.3. For the sake of simplicity, we will use the symbol $A=\left\langle x, \mu_{A}, \gamma_{A}\right\rangle$ for the IFS $A=\left\{\left\langle x, \mu_{A}(x), \gamma_{A}(x)\right\rangle: x \in X\right\}$.

EXAMPLE 2.4. Every fuzzy set $A$ on a nonempty set $X$ is obviously an IFS having the form $A=\left\{\left\langle x, \mu_{A}(x), 1-\mu_{A}(x)\right\rangle: x \in X\right\}[1]$. 
One can define several relations and operations between IFSs as follows.

DEFINITION 2.5 [2]. Let $X$ be a nonempty set, and let the IFSs $A$ and $B$ be in the form $A=\left\{\left\langle x, \mu_{A}(x), \gamma_{A}(x)\right\rangle: x \in X\right\}, B=\left\{\left\langle x, \mu_{B}(x), \gamma_{B}(x)\right\rangle: x \in X\right\}$.Then

(a) $A \subseteq_{\square} B$ if and only if for all $x \in X\left[\mu_{A}(x) \leq \mu_{B}(x)\right]$;

(b) $A \subseteq \diamond B$ if and only if for all $x \in X\left[\gamma_{A}(x) \geq \gamma_{B}(x)\right]$;

(c) $A \subseteq B$ if and only if $A \subseteq_{\square} B$ and $A \subseteq \diamond B$;

(d) $A=B$ if and only if $A \subseteq B$ and $B \subseteq A$;

(e) $A\left[B\right.$ if and only if for all $x \in X\left[\pi_{A}(x) \leq \pi_{B}(x)\right]$, where $\pi_{A}: X \rightarrow I, \pi_{A}(x)=1-$ $\mu_{A}(x)-\gamma_{A}(x)$

(f) $\bar{A}=\left\{\left\langle x, \gamma_{A}(x), \mu_{A}(x)\right\rangle: x \in X\right\}$;

(g) $A \cap B=\left\{\left\langle x, \mu_{A}(x) \wedge \mu_{B}(x), \gamma_{A}(x) \vee \gamma_{B}(x)\right\rangle: x \in X\right\}$;

(h) $A \cup B=\left\{\left\langle x, \mu_{A}(x) \vee \mu_{B}(x), \gamma_{A}(x) \wedge \gamma_{B}(x)\right\rangle: x \in X\right\}$;

(i) $\square A=\left\{\left\langle x, \mu_{A}(x), 1-\mu_{A}(x)\right\rangle: x \in X\right\}$;

(j) $\diamond A=\left\{\left\langle x, 1-\gamma_{A}(x), \gamma_{A}(x)\right\rangle: x \in X\right\}$.

We can easily generalize the operations of intersection and union in Definition 2.5 to arbitrary family of IFSs as follows.

DEFINITION 2.6. Let $\left\{A_{i}: i \in J\right\}$ be an arbitrary family of IFSs in $X$. Then

(a) $\cap A_{i}=\left\{\left\langle x, \wedge \mu_{A i}(x), \vee \gamma_{i}(x)\right\rangle: x \in X\right\}$;

(b) $\cup A_{i}=\left\{\left\langle x, \vee \mu_{A i}(x), \wedge \gamma_{A i}(x)\right\rangle: x \in X\right\}$.

DEFINITION 2.7. The ordinary complement of $A=\left\langle x, \mu_{A}, \gamma_{A}\right\rangle$ is defined by

$$
A^{C}=\left\langle x, 1-\gamma_{A}, 1-\mu_{A}\right\rangle
$$

DEFINITION 2.8. $0_{\sim}=\{\langle x, 0,1\rangle: x \in X\}$ and $1_{\sim}=\{\langle x, 1,0\rangle: x \in X\}$.

Now we will define the image and preimage of IFSs. Let $X$ and $Y$ be two nonempty sets and let $f: X \rightarrow Y$ be a function. If $B=\left\{\left\langle y, \mu_{B}(y), \gamma_{B}(y)\right\rangle: y \in Y\right\}$ is an IFS in $Y$, then the preimage of $B$ under $f$, denoted by $f^{-1}(B)$, is the IFS in $X$ defined by

$$
f^{-1}(B)=\left\{\left\langle x, f^{-1}\left(\mu_{B}\right)(x), f^{-1}\left(\gamma_{B}\right)(x)\right\rangle: x \in X\right\}
$$

Similarly, if $A=\left\{\left\langle x, \lambda_{A}(x), v_{A}(x)\right\rangle: x \in X\right\}$ is an IFS in $X$, then the image of $A$ under $f$, denoted by $f(A)$, is the IFS in $Y$ defined by $f(A)=\left\{\left\langle y, f\left(\lambda_{A}\right)(y), f\left(v_{A}\right)(y)\right\rangle: y \in Y\right\}$.

Now we generalize the concept of fuzzy topological space, first initiated by Chang [6], to the case of IFSs.

This construction, in some sense, has a close resemblance to that of Brown [5], the so-called "fuzzy ditopological space."

DEFINITION 2.9 [7]. An intuitionistic fuzzy topology (IFT) on a nonempty set $X$ is a family $\tau$ of IFSs in $X$ satisfying the following axioms:

( $\left.\mathrm{T}_{1}\right) 0_{\sim}, 1_{\sim} \in T$,

( $\left.\mathrm{T}_{2}\right) G_{1} \cap G_{2} \in \tau$ for any $G_{1}, G_{2} \in \tau$,

$\left(\mathrm{T}_{3}\right) \cup G_{i} \in \tau$, for any arbitrary family $\left\{G_{i}: i \in \tau\right\} \subseteq \tau$. 
In this case the pair $(X, \tau)$ is called an intuitionistic fuzzy topological space (IFTS) and any IFS in $\tau$ is known as an intuitionistic fuzzy open set (IFOS) in $X$.

EXAMPLE 2.10. Any fuzzy topological space $\left(X, \tau_{0}\right)$ in the sense of Chang is obviously an IFTS whenever we identify a fuzzy set $A$ in $X$ whose membership function is $\mu_{A}$ with its counterpart

$$
\left\{\left\langle x, \mu_{A}(x), 1-\mu_{A}(x)\right\rangle: x \in X\right\}
$$

as in Example 2.4.

EXAMPLE 2.11. Let $\left(X, \tau_{0}\right)$ be a fuzzy topological space in Chang's sense such that $\tau_{0}$ is not indiscrete. Suppose now that $\tau_{0}=\left\{0_{\sim}, 1_{\sim}\right\} \cup\left\{v_{i}: i \in J\right\}$. Then we can construct two IFTs on $X$ as follows:

(1) $\tau^{1}=\left\{0_{\sim}, 1_{\sim}\right\} \cup\left\{\left\langle x, v_{i}, 0\right\rangle: i \in J\right\}$,

(2) $\tau^{2}=\left\{0_{\sim}, 1_{\sim}\right\} \cup\left\{\left\langle x, 0,1-v_{i}\right\rangle: i \in J\right\}$.

EXAMPLE 2.12. Let $(X, \tau)$ be an IFTS on $X$. Then, if $\tau=\left\{G_{i}: i \in J\right\}$, where $G_{i}=$ $\left\langle x, \mu_{G i}, \gamma_{G i}\right\rangle, i \in J$, then we can also construct several IFTSs on $X$ in the following way:

(1) $\tau_{0,1}=\left\{\square G_{i}: i \in J\right\}$,

(2) $\tau_{0,2}=\left\{\diamond G_{i}: i \in J\right\}$,

(3) $\tau^{C}=\left\{G_{i}^{C}: i \in J\right\}$ (the conjugate fuzzy topology of $\tau$ ).

EXAMPLE 2.13. Let $(X, \tau)$ be an IFTS.

(a) $\tau_{1}=\left\{\mu_{G}: G \in \tau\right\}$ is a fuzzy topological space on $X$ in Chang's sense.

(b) $\tau_{2}^{*}=\left\{\gamma_{G}: G \in T\right\}$ is the family of all fuzzy closed sets of the fuzzy topological space $\tau_{2}=\left\{1-\gamma_{G}: G \in \tau\right\}$ on $X$ in Chang's sense.

(c) Since $0 \leq \mu_{A}(x)+\gamma_{A}(x) \leq 1$ for each $x \in X$ and each $G \in \tau$, we obtain $\mu_{G} \leq 1-\gamma_{G}$.

(d) Using (a) and (b) we may conclude that $\left(X, \tau_{1}, \tau_{2}\right)$ is a bifuzzy topological space.

DEFINITION 2.14. An IFTS in the sense of Lowen is a pair $\langle X, \tau\rangle$ where $\langle X, \tau\rangle$ is an IFTS and each IFS in the form $C_{\alpha, \beta}=\{\langle x, \alpha, \beta\rangle: x \in X\}$, where $\alpha, \beta \in I$ are arbitrary $(\alpha+\beta \leq 1)$, belongs to $\tau$.

Notice that any fuzzy topological space $(X, \tau)$ in the sense of Lowen is obviously an IFTS in the sense of Lowen.

DEFINITION 2.15. The complement $A$ of an IFOS $A$ in an IFTS $(X, \tau)$ is called an intuitionistic fuzzy closed set (IFCS) in $X$.

DEFINITION 2.16. Let $(X, \tau)$ be an IFTS on $X$.

(a) A subfamily $\beta \subseteq \tau$ is called a base for $(X, \tau)$, if each member of $\tau$ can be written as a union of elements in $\beta$.

(b) A subfamily $\mathbf{S} \subseteq \tau$ is called a subbase for $(X, \tau)$, if the family of all finite intersections of $\mathbf{S}$ forms a base for $(X, \tau)$. In this case it is said that $(X, \tau)$ is generated by $\mathbf{S}$.

Now we define fuzzy closure and interior operators in an IFTS. 
DeFINITION 2.17. Let $(X, \tau)$ be an IFTS and let $A=\left\{\left\langle x, \mu_{A}(x), \gamma_{A}(x)\right\rangle: x \in X\right\}$ be an IFS in $X$. Then the fuzzy interior and fuzzy closure of $A$ is defined by

$$
\begin{aligned}
\operatorname{cl}(A) & =\cap\{K: K \text { is an IFCS in } X, A \subseteq K\}, \\
\operatorname{int}(A) & =\cup\{G: G \text { is an IFOS in } X, G \subseteq A\} .
\end{aligned}
$$

It can also be shown that $\operatorname{cl}(A)$ is an IFCS and $\operatorname{int}(A)$ is an IFOS in $X$, and $A$ is an IFCS in $X$ if and only if $\operatorname{cl}(A)=A, A$ is an IFOS in $X$ if and only if $\operatorname{int}(A)=A$. In addition, for any IFS $A$ in $(X, \tau)$,

(a) $\operatorname{cl}\left(A^{-}\right)=\operatorname{int}(A)^{-}$,

(b) $\operatorname{int}\left(A^{-}\right)=\operatorname{cl}(A)^{-}$.

For further properties of fuzzy interior and closure operators you may consult [7].

Proposition 2.18. Let $(X, \tau)$ be an IFTS. If $A=\left\langle x, \mu_{A}, \gamma_{A}\right\rangle$ is an IFS in $X$, then

$$
\begin{gathered}
\operatorname{int}(A) \subseteq\left\langle x, \operatorname{int}_{\tau 1}\left(\mu_{A}\right), \mathrm{cl}_{\tau 2}\left(\gamma_{A}\right)\right\rangle \subseteq A, \\
A \subseteq\left\langle x, \mathrm{cl}_{\tau 2}\left(\mu_{A}\right), \operatorname{int}_{\tau 1}\left(\gamma_{A}\right)\right\rangle \subseteq \operatorname{cl}(A),
\end{gathered}
$$

where $\tau_{1}$ and $\tau_{2}$ are the fuzzy topological spaces on $X$ defined in Example 2.13.

Now we present the basic definitions concerning fuzzy continuity.

DEFINITION 2.19. Let $(X, \tau)$ and $(Y, \phi)$ be two IFTSs and let $f: X \rightarrow Y$ be a function. Then $f$ is said to be fuzzy continuous if and only if the preimage of each IFOS in $\phi$ is an IFOS in $\tau$.

DeFINITION 2.20. Let $(X, \tau)$ and $(Y, \phi)$ be two IFTSs and let $f: X \rightarrow Y$ be a function. Then $f$ is said to be fuzzy open if and only if the image of each IFS in $\tau$ is an IFS in $\phi$.

EXAMPLE 2.21. Let $\left(X, \tau_{0}\right),\left(X, \phi_{0}\right)$ be two fuzzy topological spaces in the sense of Chang.

(a) If $f: X \rightarrow Y$ is fuzzy continuous in the usual sense, then in this case, $f$ is fuzzy continuous in the sense of Definition 2.19, too.

(b) If $f: X \rightarrow Y$ is fuzzy open in the usual sense, then $f$ need not be fuzzy open in the sense of Definition 2.20. (If, furthermore, $f: X \rightarrow Y$ were both one-to-one and onto, then $f$ would also be fuzzy open in the sense of Definition 2.20.)

EXAMPLE 2.22. Let $(X, \tau)$ be an IFTS in the sense of Lowen, $(Y, \phi)$ an IFTS, and $c_{0} \in Y$. Then the constant function $c: X \rightarrow Y, c(x)=c_{0}$ is obviously fuzzy continuous.

EXAMPLE 2.23. Let $(Y, \phi)$ be an IFTS, $X$ a nonempty set, and $f: X \rightarrow Y$ a function. In this case $\tau=\left\{f^{-1}(H): H \in \phi\right\}$ is an IFT on $X$.

Indeed, $\tau$ is the coarsest IFT on $X$ which makes the function $f: X \rightarrow Y$ fuzzy continuous. One may call the IFT $\tau$ on $X$ the initial IFT with respect to $f$.

Proposition 2.24. Let $f:(X, \tau) \rightarrow(Y, \phi)$ be a fuzzy continuous function. Then the functions (a) $f:\left(X, \tau_{1}\right) \rightarrow\left(Y, \phi_{1}\right)$, (b) $f:\left(X, \tau_{2}\right) \rightarrow\left(Y, \phi_{2}\right)$ are also fuzzy continuous, 
where $\tau_{1}, \phi_{1}, \tau_{2}, \phi_{2}$ are the fuzzy topological spaces defined in Example 2.13. (In other words, $\tau_{1}=\left\{\mu_{G i}: G_{i} \in \tau\right\}, \phi_{1}=\left\{\lambda_{H j}: H_{j} \in \phi\right\}, \tau_{2}=\left\{1-\gamma_{G i}: G_{i} \in \tau\right\}, \phi_{2}=\left\{1-\gamma_{H j}:\right.$ $\left.H_{j} \in \phi\right\}$ if $\tau=\left\{G_{i}: i \in J\right\}, \phi=\left\{H_{j}: j \in K\right\}, G_{i}=\left\langle x, \mu_{G i}, \gamma_{G i}\right\rangle$ and $\left.H_{j}=\left\langle x, \lambda_{H j}, \gamma_{H j}\right\rangle.\right)$

The proof of Proposition 2.24 follows immediately from the definitions.

3. Intuitionistic fuzzy compactness. First we present the basic concepts.

DEFINITION 3.1 [7]. Let $(X, \tau)$ be an IFTS.

(a) If a family $\left\{\left\langle x, \mu_{G i}, \gamma_{G i}\right\rangle: i \in J\right\}$ of IFOSs in $X$ satisfies the condition $\cup\left\{\left\langle x, \mu_{G i}\right.\right.$, $\left.\left.\gamma_{G i}\right\rangle: i \in J\right\}=1_{\sim}$, then it is called a fuzzy open cover of $X$. A finite subfamily of a fuzzy open cover $\left\{\left\langle x, \mu_{G i}, \gamma_{G i}\right\rangle: i \in J\right\}$ of $X$, which is also a fuzzy open cover of $X$, is called a finite subcover of $\left\{\left\langle x, \mu_{G i}, \gamma_{G i}\right\rangle: i \in J\right\}$.

(b) A family $\left\{\left\langle x, \mu_{K i}, \gamma_{K i}\right\rangle: i \in J\right\}$ of IFCSs in $X$ satisfies the finite intersection property (FIP) if and only if every finite subfamily $\left\{\left\langle x, \mu_{K i}, \gamma_{K i}\right\rangle: i=1,2,3, \ldots, n\right\}$ of the family satisfies the condition $\cap\left\langle x, \mu_{K i}, \gamma_{K i}\right\rangle \neq 0 \sim$.

(c) An IFTS $(X, \tau)$ is called fuzzy compact if and only if every fuzzy open cover of $X$ has a finite subcover.

Now we will give two characterizations of fuzzy compactness.

Proposition 3.2. An IFTS $(X, \tau)$ is fuzzy compact if and only if for each family $\delta=\left\{G_{i}: i \in J\right\}$, where $G_{i}=\left\langle x, \mu_{G i}, \gamma_{G i}\right\rangle(i \in J)$ of IFOSs in $X$ with the properties $\vee \mu_{G i}=1$ and $\vee\left(1-\gamma_{G i}\right)=1$, there exists a finite subfamily $\left\{G_{i}: i=1,2, \ldots, n\right\}$ of $\delta$ such that $\vee_{i=1}^{n} \mu_{G i}=1$ and $\vee_{i=1}^{n}\left(1-\gamma_{G i}\right)=1$.

The proof of Proposition 3.2 is obvious.

Proposition 3.3. An IFTS $(X, \tau)$ is fuzzy compact if and only if every family $\left\{\left\langle x, \mu_{K i}\right.\right.$, $\left.\left.\gamma_{K i}\right\rangle: i \in J\right\}$ of IFCSs on $X$ having the FIP has a nonempty intersection.

For the proof of Proposition 3.3 see [7]. Here we state that fuzzy compactness is preserved under a fuzzy continuous bijection.

Proposition 3.4. Let $(X, \tau),(Y, \phi)$ be IFTSs and $f: X \rightarrow Y$ a fuzzy continuous bijection. If $(X, \tau)$ is fuzzy compact, then so is $(Y, \phi)$.

For the proof of Proposition 3.4 see [7].

DEFINITION 3.5 [7]. Let $(X, \tau)$ be an IFTS and $A$ an IFS in $X$.

(a) If a family $\left\{\left\langle x, \mu_{G i}, \gamma_{G i}\right\rangle: i \in J\right\}$ of IFOSs in $X$ satisfies the condition $A \subseteq \cup\{\langle x$, $\left.\left.\mu_{G i}, \gamma_{G i}\right\rangle: i \in J\right\}$, then it is called a fuzzy open over of $A$. A finite subfamily of the fuzzy open cover $\left\{\left\langle x, \mu_{G i}, \gamma_{G i}\right\rangle: i \in J\right\}$ of $A$, which is also a fuzzy open cover of $A$, is called a finite subcover of $\left\{\left\langle x, \mu_{G i}, \gamma_{G i}\right\rangle: i \in J\right\}$.

(b) An IFS $A=\left\langle x, \mu_{A}, \gamma_{A}\right\rangle$ in an IFTS $(X, \tau)$ is called fuzzy compact if and only if every fuzzy open cover of $A$ has a finite subcover.

COROLlary 3.6 [7]. An IFS $A=\left\langle x, \mu_{A}, \gamma_{A}\right\rangle$ in an IFTS $(X, \tau)$ is fuzzy compact if and only if for each family $\delta=\left\{G_{i}: i \in J\right\}$, where $G_{i}=\left\langle x, \mu_{G i}, \gamma_{G i}\right\rangle$ ( $i \in J$ ) of IFOSs in $X$ with the properties $\mu_{A} \subseteq \vee_{i \in J} \mu_{G i}$ and $1-\gamma_{A} \subseteq \vee_{i \in J}\left(1-\gamma_{G i}\right)$, there exists a finite subfamily 
$\left\{G_{i}: i=1,2, \ldots, n\right\}$ of $\delta$ such that

$$
\mu_{A} \subseteq \vee_{i=1}^{n} \mu_{G i}, \quad 1-\gamma_{A} \subseteq \vee_{i=1}^{n}\left(1-\gamma_{G i}\right)
$$

EXAMPLE 3.7. Let $\left(X, \tau_{0}\right)$ be a fuzzy topological space in Chang's sense and $\mu_{A} \in I^{x}$ a fuzzy compact set in $X$. We can construct an IFTS $\tau$ on $X$ as in [7, Example 5.9]. Now the IFS $A=\left\langle x, \mu_{A}, 1-\mu_{A}\right\rangle$, also fuzzy compact in the $\operatorname{IFTS}(X, \tau)$.

Corollary 3.8. Let $(X, \tau),(Y, \phi)$ be IFTSs and $f: X \rightarrow Y$ a fuzzy continuous bijection. If $A$ is fuzzy compact in $(X, \tau)$, then so is $f(A)$ in $(Y, \phi)$.

For the proof of Corollary 3.8 see [7]. Here we present a version of the Alexander subbase lemma for IFTSs.

Proposition 3.9 (the Alexander subbase lemma). Let $\delta$ be a subbase of an IFTS $(X, \tau)$. Then $(X, \tau)$ is fuzzy compact if and only if for each family of IFCSs chosen from $\delta^{c}=\{K: K \in \delta\}$ having the FIP there is a nonzero intersection.

Proof. Let $K=\left\langle x, \mu_{K}, \gamma_{K}\right\rangle$ denote a typical element in $\delta^{c}$.

“IF” PART. Straightforward.

“ONLY IF" PART. Suppose, on the contrary, that $(X, \tau)$ is not fuzzy compact. Then there exists a family $K=\left\{\left\langle x, \mu_{K i}, \gamma_{K i}\right\rangle: i \in J\right\}$ of IFCSs in $X$ having the FIP such that $\cap_{i \in J} K_{i}=0_{\sim}$. The collection of all such families $K$, ordered by classical inclusion $(\subseteq)$, is inductive. Hence, by Zorn's lemma, we may find a maximal family $K$, say $M=\left\{M_{j}: j \in\right.$ $K$, where $M_{j}=\left\langle x, \mu_{M j}, \gamma_{M j}\right\rangle, j \in K$. The family $M$ satisfies the following properties:

(1) $M$ is a fuzzy filter consisting of IFCSs in $(X, \tau)$,

(2) $\cap\left(M \cap \delta^{c}\right)=0$.

Now the second assertion gives an immediate contradiction to the hypothesis of the theorem. Hence, $(X, \tau)$ is fuzzy compact.

4. A Tychonoff theorem in IFTSs. With a Tychonoff-like theorem in mind, we must first present the product of IFTSs. Let $\left(X_{i}, \tau_{i}\right)$ be an IFTS on $X_{i}$ for each $i \in J$, and let $X=\pi X_{i}$. For each $i \in J$, we may construct the $i$ th projection mapping as follows:

$$
\pi_{i}: X \longrightarrow X_{i}, \quad \pi_{i}\left(\left(x_{j}\right)_{j \in J}\right)=x_{i}
$$

Then we define

$$
\mathbf{S}=\left\{\pi_{i}^{-1}\left(S_{i}\right): i \in J, S_{i} \in \tau_{i}\right\}
$$

DEFINITION 4.1. The product set $X$ equipped with the IFT generated on $X$ by the family $\mathbf{S}$ is called the product of the IFTSs $\left\{\left(X_{i}, \boldsymbol{T}_{i}\right): i \in J\right\}$.

For each $i \in J$ and for each $S_{i} \in \tau_{i}$ we have $\pi_{i}^{-1}\left(S_{i}\right) \in \tau$; so $\pi_{i}$ is indeed a fuzzy continuous function from the product IFTS onto $\left(X_{i}, \tau_{i}\right)$ for all $i \in J$. The product IFT $\tau$ is, of course, the coarsest IFT on $X$ having this property. 
Now we express Tychonoff theorem in two steps, one of which is valid in general, but its reverse is true for finitely many terms.

THEOREM 4.2. For each $i \in J$ let $\left(X_{i}, \tau_{i}\right)$ be an IFTS on $X_{i}$. Then if the product IFTS $(X, \tau)$ is fuzzy compact, then each $\left(X_{i}, \tau_{i}\right)$ is fuzzy compact.

The proof of Theorem 4.2 follows from Corollary 3.8 .

THEOREM 4.3. Let the IFTSS $\left(X_{1}, \tau_{1}\right)$ and $\left(X_{2}, \tau_{2}\right)$ be fuzzy compact. Then the product IFTS on $X=X_{1} \times X_{2}$ is fuzzy compact, too.

Proof. Here we will make use of the Alexander subbase lemma. Suppose, on the contrary, that there exists a family

$$
P=\left\{\pi_{1}^{-1}\left(P_{i 1}\right): i_{1} \in J_{1}\right\} \cup\left\{\pi_{2}^{-1}\left(P_{i 2}\right): i_{2} \in J_{2}\right\}
$$

consisting of some of the IFCSs obtained from the subbase

$$
\delta=\left\{\pi_{1}^{-1}\left(T_{1}\right), \pi_{2}^{-1}\left(T_{2}\right): T_{1} \in \tau_{1}, T_{2} \in T_{2}\right\}
$$

of the product IFT on $X$ such that $P$ has the FIP and $\cap P=0$. Now it can be shown easily that the families

$$
P_{1}=\left\{P_{i 1}: i_{1} \in J_{1}\right\}, \quad P_{2}=\left\{P_{i 2}: i_{2} \in J_{2}\right\}
$$

have the FIP, and since $\left(X_{i}, \tau_{i}\right)$ 's $(i=1,2)$ are fuzzy compact, we have $\cap P_{1} \neq 0$ and $\cap P_{2} \neq 0$ which mean that

$$
\left(\wedge \mu_{P i 1} \neq 0 \text { or } \vee \gamma_{P i 1} \neq 1\right), \quad\left(\wedge \mu_{P i 2} \neq 0 \text { or } \vee \gamma_{P i 2} \neq 1\right)
$$

But from $\cap P=0$ we obtain

$$
\left(\wedge \mu_{P i 1} \circ \pi_{1}\right) \wedge\left(\wedge \mu_{P i 2} \circ \pi_{2}\right)=0, \quad\left(\vee \gamma_{P i 1} \circ \pi_{1}\right) \vee\left(\vee \gamma_{P i 2} \circ \pi_{2}\right)=1 .
$$

Here there exist four cases.

CASE 1. If $\wedge \mu_{P i 1} \neq 0$ and $\wedge \mu_{P i 2} \neq 0$, then there exist $x_{1} \in X_{1}, x_{2} \in X_{2}$ such that $\wedge \mu_{P i 1}\left(x_{1}\right) \neq 0$ and $\wedge \mu_{P i 2}\left(x_{2}\right) \neq 0$ from which we obtain a contradiction to (4.7), if it is evaluated in $\left(x_{1}, x_{2}\right)$.

CASE 2. If $\vee \gamma_{P i 1} \neq 1$ and $\vee \gamma_{P i 2} \neq 1$, then we get a similar contradiction as in the first case.

CASE 3. If $\wedge \mu_{P i 1} \neq 0$ and $\vee \gamma_{P i 2} \neq 1$, then there exist $x_{1} \in X_{1}, x_{2} \in X_{2}$ such that $\wedge \mu_{P i 1}\left(x_{1}\right) \neq 0$ and $\vee \gamma_{P i 2}\left(x_{2}\right) \neq 1$ from which we obtain $\wedge \mu_{P i 2}\left(x_{2}\right)=0$ and $\vee \gamma_{P i 1}\left(x_{1}\right)=$ 1 , and then, since $\gamma_{P i 1} \leq 1-\mu_{P i 1}$ for each $P_{i 1}$,

$$
\begin{aligned}
& \vee \gamma_{P i 1} \leq\left(1-\mu_{P i 1}\right)=1-\wedge \mu_{P i 1} \\
& \Longrightarrow 1=\vee \gamma_{P i 1}\left(x_{1}\right) \leq 1-\wedge \mu_{P i 1}\left(x_{1}\right) \\
& \Longrightarrow \wedge \mu_{P i 1}\left(x_{1}\right)=0,
\end{aligned}
$$

we obtain a contradiction. 
CASE 4. If $\vee \gamma_{P i 1} \neq 1$ and $\wedge \mu_{P i 2} \neq 0$, then we obtain a similar contradiction as in the third case.

Hence, by the Alexander subbase lemma, $(X, \tau)$ is also fuzzy compact.

5. Intuitionistic fuzzy dicompactness. First we present a new kind of compactness in IFTSs.

DeFINITION 5.1. Let $(X, \tau)$ be an IFTS $K=\left\{K_{i}: i \in J\right\}$, where $K_{i}=\left\langle x, \mu_{K i}, \gamma_{K i}\right\rangle, i \neq J$, is a family of IFCSs in $X$. Then

(a) $K$ is said to have the finite exclusion property (FEP) if for each finitely many $K_{1}, K_{2}, \ldots, K_{n}$ chosen from $K$ there exists $\wedge \mu_{K i} \leq \vee \gamma_{K i}$,

(b) $K$ is said to be bound in $(X, \tau)$ if $\wedge \mu_{K i} \leq \vee \gamma_{K i}$.

DEFINITION 5.2. An IFTS $(X, \tau)$ is called fuzzy dicompact if and only if every family $K$ of IFCSs in $(X, \tau)$ is bound in $(X, \tau)$.

Proposition 5.3 (the Alexander subbase lemma). Let $\delta$ be a subbase of an IFTS $(X, \tau)$. Then $(X, \tau)$ is fuzzy dicompact if and only if each family of IFCSs chosen from $\delta^{c}=\left\{G^{-}: G \in \tau\right\}$ having the FEP is bound in $(X, \tau)$.

Proof. Let $G=\left\langle x, \cdot \mu_{G}, \gamma_{G}\right\rangle$ denote a typical element in $\delta$.

"IF" PART. This is straightforward.

“ONLY IF" PART. Suppose, on the contrary, that $(X, \tau)$ is not fuzzy dicompact. Then there exists a family $K=\left\{\left\langle x, \mu_{K i}, \gamma_{K i}\right\rangle: i \in J\right\}$ of IFCSs in $X$ having the FEP such that $\wedge_{i \in j} \mu_{K i} \leq \vee_{i \in J} \gamma_{K i}$.

The collection of all such families $K$, ordered by classical inclusion $(\subseteq)$, is inductive. Hence, by Zorn's lemma, we may find a maximal family $K$, say $M=\left\{\left\langle x, \mu_{M i}, \gamma_{M i}\right\rangle: i \in J\right\}$. The family $M$ satisfies the following properties:

(1) $\mathbf{F}=\left\{\gamma_{M i}: i \in J\right\}$ is a fuzzy filter in $\left(X, \tau_{2}\right)$,

(2) $\mathbf{G}=\left\{\mu_{M i}: i \in J\right\}$ is a fuzzy filter in $\left(X, \tau_{1}\right)$,

(3) $\wedge\left(\mathbf{F} \cap\left\{\gamma_{G}: G \in \delta\right\}\right) \leq \vee\left(\mathbf{G} \cap\left\{\mu_{G}: G \in \delta\right\}\right)$.

Now the third assertion gives an immediate contradiction to the hypothesis of the theorem. Hence, $(X, \tau)$ is fuzzy dicompact.

Here we state that fuzzy dicompactness is preserved under a fuzzy continuous bijection.

Proposition 5.4. Let $(X, \tau),(Y, \phi)$ be IFTSs and $f: X \rightarrow Y$ a fuzzy continuous bijection. If $(X, \tau)$ is fuzzy dicompact, then so is $(Y, \phi)$.

Proof. Let $K=\left\{K_{i}: i \in J\right\}$, where $K_{i}=\left\langle y, \mu_{K i}, \gamma_{K i}\right\rangle, i \in J$, be a family of IFCSs in $(Y, \phi)$ with the FEP. Now define

$$
f^{-1}(K)=\left\{f^{-1}\left(K_{i}\right): i \in J\right\}
$$

Then $f^{-1}(K)$ consists of IFCSs in $(X, \tau)$ since $f$ is fuzzy continuous, and furthermore it has the FEP. (Suppose that it does not have the FEP. Hence there exist $i=1,2, \ldots, n$ 
such that

$$
\wedge_{i=1}^{n} f^{-1}\left(\mu_{K i}\right) \leq \vee_{i=1}^{n} f^{-1}\left(\gamma_{K i}\right) .
$$

Hence $f\left(\wedge_{i=1}^{n} f^{-1}\left(\mu_{K i}\right)\right) \leq f\left(\vee_{i=1}^{n} f^{-1}\left(\gamma_{K i}\right)\right)$ implies $\wedge_{i=1}^{n} f\left(f^{-1}\left(\mu_{K i}\right)\right) \leq \vee_{i=1}^{n} f\left(f^{-1}\left(\gamma_{K i}\right)\right)$. (Since $f$ is $1-1$.) This implies

$$
\wedge_{i=1}^{n} \mu_{K i} \leq \vee_{i=1}^{n} \gamma_{K i} \quad \text { (since } f \text { is onto) }
$$

which is in contradiction with the hypothesis.) Since $(X, \tau)$ is fuzzy dicompact, we have $\wedge_{i \in j} f^{-1}\left(\mu_{K i}\right) \leq \vee_{i \in j} f^{-1}\left(\gamma_{K i}\right)$ from which we obtain $f^{-1}\left(\wedge_{i \in j} \mu_{K i}\right)=\wedge_{i \in j} f^{-1}\left(\mu_{K i}\right)$ $\leq \vee_{i \in j} f^{-1}\left(\gamma_{K i}\right)=f^{-1}\left(\vee_{i \in j} \gamma_{K i}\right)$ implies $\wedge_{i \in j} f^{-1}\left(\mu_{K i}\right) \leq\left(\vee_{i \in j} \gamma_{K i}\right)$, which is an obvious contradiction.

Hence $(Y, \phi)$ is fuzzy dicompact, too.

\section{REFERENCES}

[1] K. T. Atanassov, Intuitionistic fuzzy sets, VII ITKR's Session, Sofia, 1983.

[2] _ Review and new results on intuitionistic fuzzy sets, Mathematical Foundations of Artificial Intelligence Seminar (Sofia), 1988, pp. 1-8.

[3] K. T. Atanassov and S. Stoeva, Intuitionistic fuzzy sets, Proc. Polish Symposium on Interval and Fuzzy Mathematics (Poznan), 1983, pp. 23-26.

[4] _ Intuitionistic L-fuzzy sets, Cybernetics and Systems Research 2, Elsevier Science Publishers, Amsterdam, 1984, pp. 539-540.

[5] L. M. Brown, Fuzzy ditopological spaces, Proc. 2nd International Conference of the Balkanic Union for Fuzzy Systems and Artificial Intelligence (BUFSA), Karadeniz Technical University, Trabzon, 1992.

[6] C. L. Chang, Fuzzy topological spaces, J. Math. Anal. Appl. 24 (1968), 182-190.

[7] D. Çoker, An introduction to intuitionistic fuzzy topological spaces, Fuzzy Sets and Systems 88 (1997), no. 1, 81-89.

[8] L. A. Zadeh, Fuzzy sets, Information and Control 8 (1965), 338-353.

Doğan Çoker: Department of Mathematics, Hacettepe University, 06532 Beytepe-Ankara, Turkey

A. Haydar Eş: Department of Mathematics, Hacettepe University, 06532 Beytepe-Ankara, Turkey E-mail address: ahaydar@hacettepe.edu.tr

Necla Turanli: Department of Mathematics Education, Hacettepe University, 06532 BeytepeAnkara, Turkey

E-mail address: turan7i@anacettepe.edu.tr 


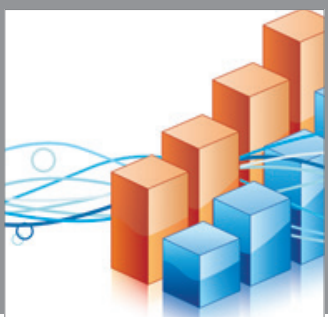

Advances in

Operations Research

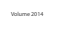

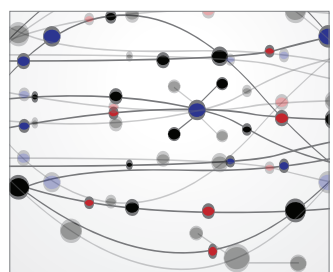

\section{The Scientific} World Journal
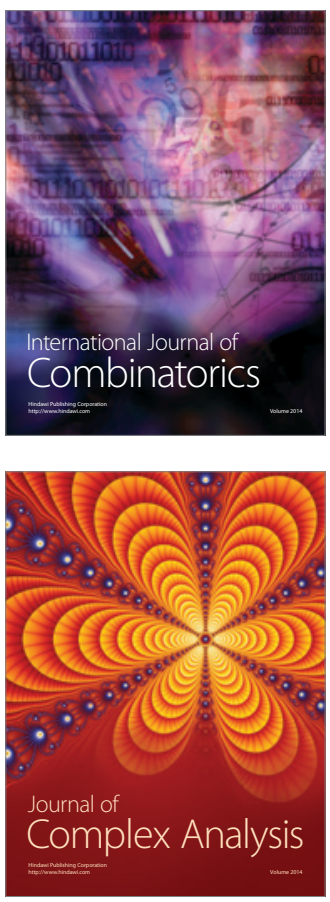

International Journal of

Mathematics and

Mathematical

Sciences
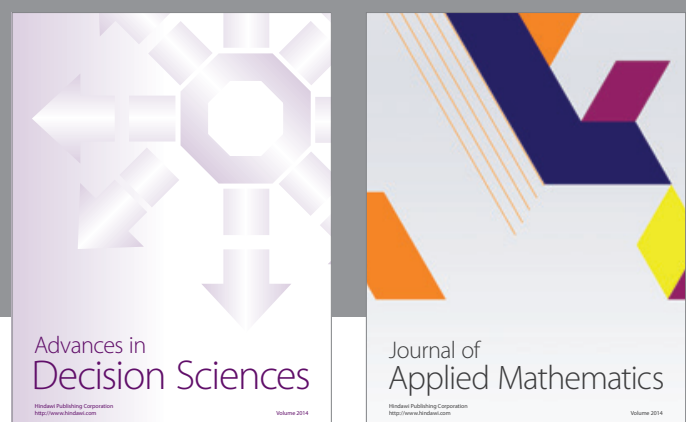

Journal of

Applied Mathematics
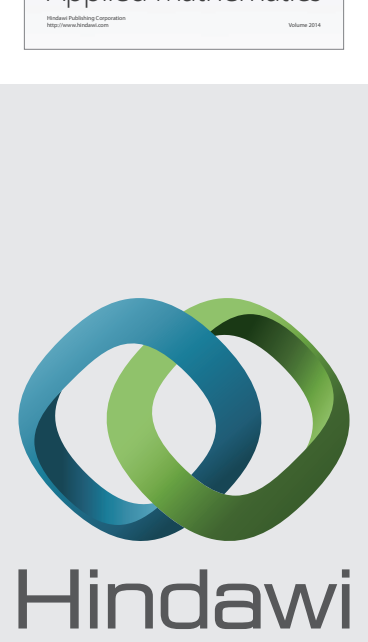

Submit your manuscripts at http://www.hindawi.com
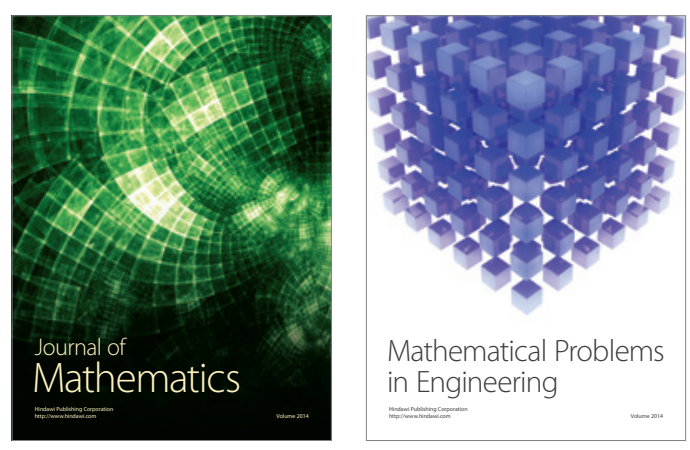

Mathematical Problems in Engineering
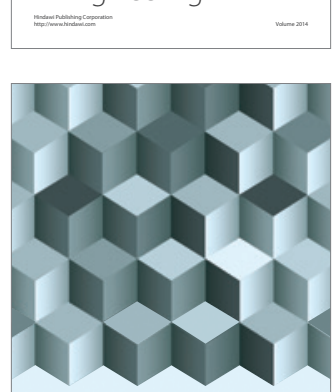

Journal of

Function Spaces
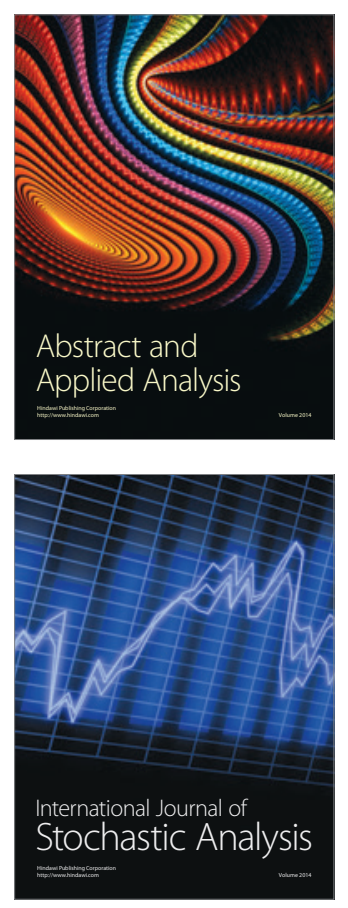

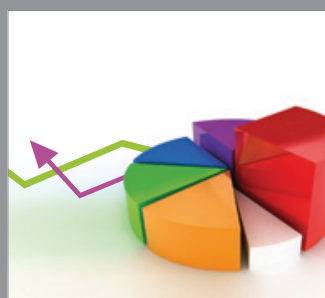

ournal of

Probability and Statistics

Promensencen
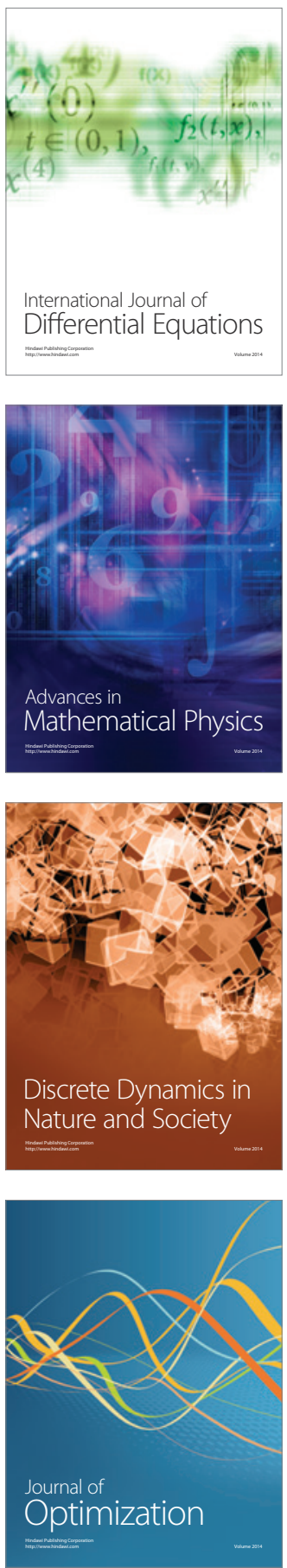